\section{A FISIOTERAPIA NA ATENÇÃo PRIMÁRIA À SAÚDE: UMA REVISÃO INTEGRATIVA}

\author{
Physical therapy in primary health care: an integrative review \\ La fisioterapia en la atención primaria de salud: una revisión \\ integrativa
}

\section{RESUMO}

Objetivo: Analisar as atividades desenvolvidas pela fisioterapia na atenção primária à saúde. Métodos: Revisão integrativa da literatura realizada nas bases SciELO, LILACS e MEDLINE usando os descritores physiotherapy, physical therapy e primary health care, em maio e junho de 2015. Buscaram-se artigos sobre a atividade do fisioterapeuta na atenção primária, disponíveis na íntegra, em inglês, português ou espanhol sem restrição de ano de publicação. Os artigos foram selecionados e analisados mediante as seguintes variáveis: atividades desenvolvidas, principais resultados e dificuldades enfrentadas. Resultados: Sete artigos foram analisados. As atividades enfatizaram atenção individual e coletiva tanto em nível preventivo quanto de reabilitação junto a diferentes públicos e apresentaram resultados satisfatórios com impacto positivo na saúde e redução de custos individuais e coletivos. No entanto, enfrentam desafios como o número insuficiente de profissionais, a falta de recursos e infraestrutura, a dificuldade de trabalho em equipe e a necessidade de mudanças na formação profissional. Conclusão: As atividades, apesar de incipientes e dos entraves enfrentados, apresentam bons resultados. Tal constatação demonstra a importância da fisioterapia na atenção primária à saúde e contribui para a difusão e efetiva atuação do profissional nesse nível de atenção.

Descritores: Fisioterapia; Atenção Primária à Saúde; Saúde Pública.

\section{ABSTRACT}

Objective: To analyze physical therapy activities carried out in primary health care. Method: Integrative literature review carried out in SciELO, LILACS and MEDLINE databases using the descriptors physiotherapy, physical therapy and primary health care in May and June 2015. We searched for articles on the physical therapist's work in primary care fully available in English, Portuguese or Spanish regardless of the year of publication. The articles were selected and analyzed based on the following variables: activities developed, main results and the difficulties faced. Results: Seven articles were analyzed. Activities focused on individual and collective care, both at the preventive and rehabilitation levels, of different populations and showed satisfactory results with a positive impact on health and reduced individual and collective costs. However, they face challenges such as the insufficient number of professionals, lack of resources and infrastructure, teamwork problems, and the need for changes in vocational training. Conclusion: The activities, despite being incipient and facing obstacles, present good results. This finding demonstrates the importance of physical therapy in primary health care and contributes to the dissemination and effective performance of the professional at this level of care.

Descriptors: Physical Therapy Specialty; Primary Health Care; Public Health.
Artigo de Revisão
1) Universidade Federal do Piauí - UFPI Teresina (PI) - Brasil

Recebido em: 10/04/2016

Revisado em: 13/05/2016

Aceito em: 25/06/2016 


\section{RESUMEN}

Objetivo: Analizar las actividades desarrolladas por la fisioterapia en la atención primaria de salud. Métodos: Revisión integrativa de la literatura realizada en las bases de datos SciELO, LILACS y MEDLINE utilizando los descriptores physiotherapy, physical therapy y primary health care entre mayo y junio de 2015. Se buscó artículos sobre la actividad del fisioterapeuta en la atención primaria, disponibles en su totalidad en los idiomas inglés, portugués o español sin restricción del año de publicación. Los artículos fueron elegidos y analizados mediante las siguientes variables: actividades desarrolladas, principales resultados y dificultades afrontadas. Resultados: Siete artículos fueron analizados. Las actividades enfatizaron la atención individual y colectiva en nivel de prevención $y$ rehabilitación junto a públicos distintos y presentaron resultados satisfactorios con impacto positivo en la salud y reducción de costes individuales y colectivos. Sin embargo, hay desafios como el número insuficiente de profesionales, la falta de recursos e infraestructura, la dificultad de trabajo en equipo y la necesidad de cambios en la formación profesional. Conclusión: A pesar de las trabas afrontadas y aunque sean actividades incipientes, las mismas presentan buenos resultados. Tales resultados demuestran la importancia de la fisioterapia en la atención primaria de salud y contribuye para la difusión y actuación efectiva del profesional de ese nivel de atención.

Descriptores: Fisioterapia; Atención Primaria de Salud; Salud Pública.

\section{INTRODUÇÃO}

No Brasil, com a implantação do Sistema Único de Saúde (SUS) o modelo de atenção à saúde foi reformulado inserindo a atenção primária com o objetivo de reorientar o sistema e valorizar ações de promoção de saúde e prevenção de $\operatorname{agravos}^{(1)}$.

A fim de fortalecer esse modelo de atenção foi assumida pelo Ministério da Saúde em 1994, a Estratégia Saúde da Família (ESF) na qual, por meio de assistência integral e multiprofissional, centrada na comunidade, a atenção primária tem reafirmada sua inserção na rede de saúde. Mais recentemente, em 2008, surgiram os Núcleos de Apoio à Saúde da Família (NASF) que têm por objetivo prestar auxílio às equipes da ESF e ampliar a oferta do cuidado no nível primário reafirmando a integralidade, qualidade e resolubilidade do sistema ${ }^{(2,3)}$.

A fisioterapia como potencial integrante da equipe multidisciplinar do NASF também necessita reformular-se de modo que a reabilitação dedicada ao seu objeto de estudo, compartilhe espaço com atividades de promoção, educação em saúde e prevenção de riscos de modo a desvincular-se do paradigma de assistência restrita ao nível de atenção terciária e aproximar-se da atenção primária ${ }^{(2,4-7)}$.

Dessa forma, faz-se necessário o conhecimento das ações desenvolvidas pela fisioterapia no âmbito da atenção primária a fim de elucidar como está sendo construída sua inserção no nível primário bem como difundir este campo de atuação do profissional. Nesse sentido, objetivou-se analisar as atividades desenvolvidas pela fisioterapia na atenção primária à saúde.

\section{MÉTODOS}

Trata-se de uma revisão integrativa da literatura desenvolvida a partir da questão norteadora: Quais atividades são desenvolvidas pela fisioterapia na atenção primária à saúde?

Para desenvolvimento do estudo, foram seguidas as etapas: seleção da questão norteadora; estabelecimento de palavras-chave, critérios de inclusão e exclusão e busca na literatura; avaliação dos estudos incluídos na revisão integrativa; definição das informações a serem extraídas; interpretação dos resultados e apresentação de síntese do conhecimento produzido ${ }^{(8)}$.

As buscas foram realizadas nas bases de dados SciELO (Scientific Eletronic Library Online), LILACS (Literatura Latino-Americana e do Caribe em Ciências da Saúde) e MEDLINE (National Library of Medicine), mediante os descritores fisioterapia (physiotherapy, physical therapy) e atenção primária à saúde (primary health care) de acordo como a terminologia em saúde DeCS, (Descritores em Ciências da Saúde) da Biblioteca Virtual em Saúde. Para sistematizar as buscas foram utilizados os operadores booleanos com o seguinte esquema: (physiotherapy OR physical therapy AND primary health care).

Os critérios de elegibilidade foram: artigos sobre atividade do fisioterapeuta na atenção primária à saúde, disponíveis na íntegra, em língua inglesa, portuguesa ou espanhola, sem limitação de ano de publicação. Foram excluídas revisões da literatura, teses e monografias, propostas de atuação ou trabalhos que descreviam atividades acadêmicas.

A busca foi realizada nos meses de maio e junho de 2015, por duas pesquisadoras, de forma independente.

\section{RESULTADOS}

Inicialmente foram encontrados 315 artigos. Após leitura de títulos, resumos e classificação pelos critérios de inclusão foram selecionados 7 artigos. A busca e seleção dos trabalhos são apresentadas conforme o instrumento PRISMA (Preferred Reporting Items for Systematic Review and Meta-Analyses) (Figura 1). 


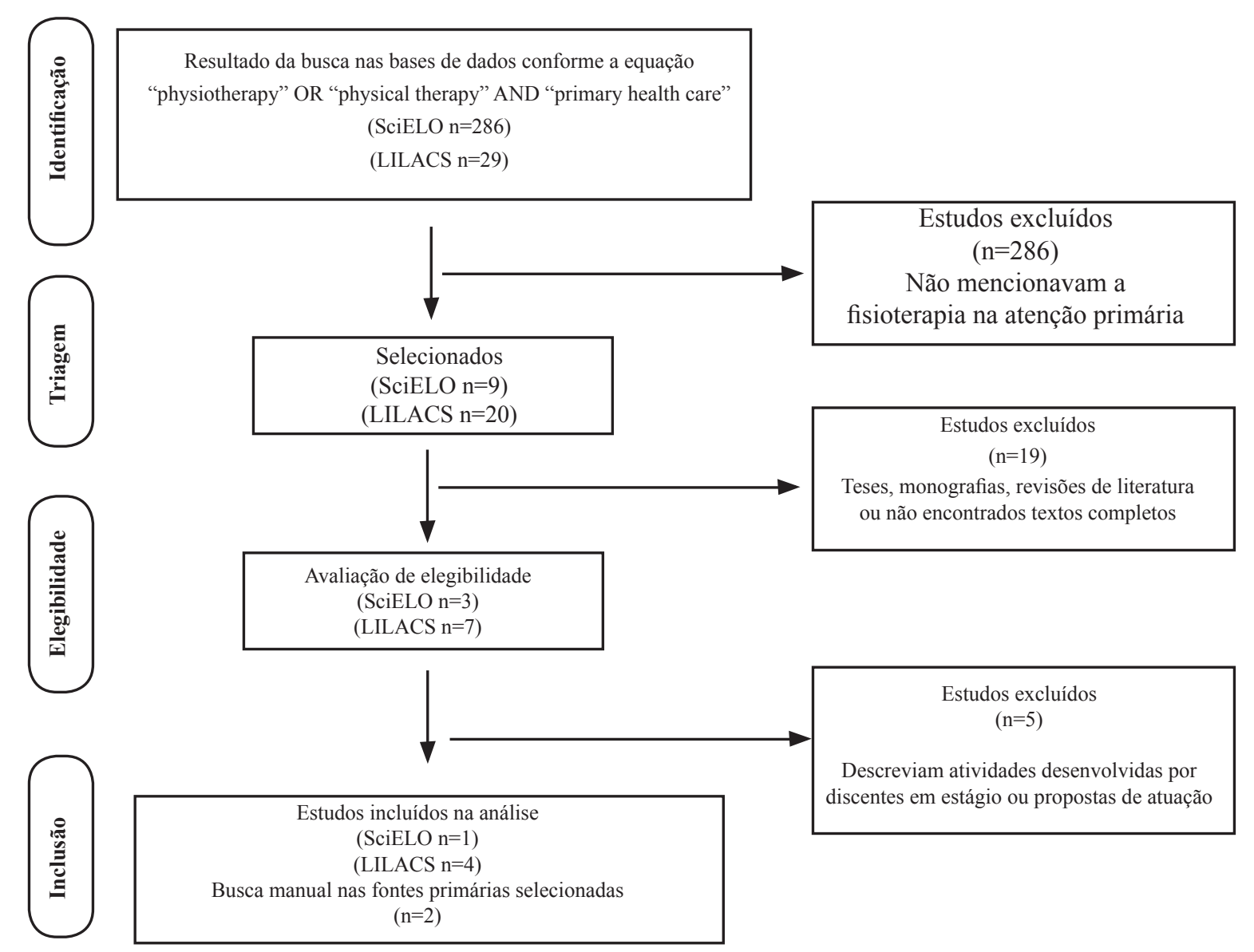

Figura 1 - Fluxograma de seleção dos estudos. Teresina, PI, Brasil, 2015.

Quadro I - Caracterização dos estudos selecionados. Teresina, PI, Brasil, 2015.

\begin{tabular}{|c|c|c|c|c|c|}
\hline Autores & Ano & Local & Equipe & $\mathrm{n}$ & Público-alvo \\
\hline Dibai Filho, Aveiro $^{(5)}$ & 2012 & Arapiraca, AL & NASF & 8 & Idosos \\
\hline $\begin{array}{l}\text { Trelha, Silva, Lida, Fortes, } \\
\text { Mendes }^{(10)}\end{array}$ & 2007 & Londrina, PR & PSF & 4 & Predominância de pacientes acamados. \\
\hline Rocha, Kretzer ${ }^{(11)}$ & 2009 & São Paulo, SP & ESF & 3 & Portadores de deficiência ou incapacidade. \\
\hline $\begin{array}{l}\text { Brasil, Brandão, Silva, } \\
\text { Gondim Filho }^{(12)}\end{array}$ & 2005 & Sobral, CE & PSF & 4 & $\begin{array}{l}\text { Indivíduos com diversas condições desde } \\
\text { neurológicas a gineco-obstétricas. }\end{array}$ \\
\hline Yonamine, Trelha ${ }^{(13)}$ & 2009 & Londrina, PR & $\begin{array}{c}\text { USF } \\
\text { RMSFC }\end{array}$ & 1 & $\begin{array}{l}\text { Predominância de indivíduos com agravos } \\
\text { osteomusculares e respiratórios. }\end{array}$ \\
\hline $\begin{array}{l}\text { Langoni, Valmorbida, } \\
\text { Resende }^{(14)}\end{array}$ & 2012 & Porto Alegre, RS & $\begin{array}{l}\text { UAPS } \\
\text { RMSFC }\end{array}$ & 2 & $\begin{array}{l}\text { Usuários em acompanhamento, com } \\
\text { dificuldade de locomoção, atraso no } \\
\text { desenvolvimento neuropsicomotor, } \\
\text { alterações osteomusculares, incontinência } \\
\text { urinária ou agravo respiratório. }\end{array}$ \\
\hline $\begin{array}{l}\text { Figueiredo, Baracho, Vaz, } \\
\text { Sampaio }^{(15)}\end{array}$ & 2012 & Belo Horizonte, MG & UBS & 2 & $\begin{array}{l}\text { Funcionárias de unidade básica de saúde } \\
\text { e pacientes com queixa de incontinência } \\
\text { urinária. }\end{array}$ \\
\hline
\end{tabular}

n: Número de fisioterapeutas atuantes nas equipes avaliadas. CE: Ceará. PSF: Programa Saúde da Família. PR: Paraná. USF: Unidade de Saúde da Família. RMSFC: Residência Multiprofissional em Saúde da Família e Comunidade. SP: São Paulo. ESF: Estratégia Saúde da Família. AL: Alagoas. NASF: Núcleo de Apoio à Saúde da Família. RS: Rio Grande do Sul. UAPS: Unidade de Atenção Primária à Saúde. MG: Minas Gerais. UBS: Unidade Básica de Saúde. 
Quadro II - Atividades desenvolvidas pela fisioterapia na atenção primária a saúde. Teresina, PI, Brasil, 2015.

\begin{tabular}{|c|c|c|c|}
\hline Autor/Ano & Atividades desenvolvidas & Principais resultados & Dificuldades \\
\hline $\begin{array}{l}\text { Dibai Filho, } \\
\text { Aveiro, } \\
2012^{(5)}\end{array}$ & $\begin{array}{l}\text { Ênfase em ações educativas, } \\
\text { preventivas e promotoras de saúde com } \\
\text { palestras, atividades em grupo; visitas } \\
\text { domiciliares; encaminhamento para } \\
\text { serviços de referência. }\end{array}$ & $\begin{array}{c}\text { Avaliado como positivo o cuidado ao } \\
\text { idoso no NASF. Porém, apesar das ações } \\
\text { educativas e preventivas, na prática, } \\
\text { ainda persistem ações convencionais, } \\
\text { que entendem o idoso como um } \\
\text { indivíduo doente e voltam-se à terapia } \\
\text { medicamentosa. }\end{array}$ & $\begin{array}{c}\text { Capacitação, materiais e } \\
\text { infraestrutura }\end{array}$ \\
\hline $\begin{array}{l}\text { Trelha, Silva, } \\
\text { Lida, Fortes, } \\
\text { Mendes, } \\
2007^{(10)}\end{array}$ & $\begin{array}{l}\text { Atendimentos individuais, em grupos e } \\
\text { visitas domiciliares com orientações a } \\
\text { cuidadores; encaminhamento a serviços } \\
\text { especializados. Atividades preventivas e } \\
\text { educativas por meio de palestras. }\end{array}$ & $\begin{array}{c}\text { As entrevistadas ressaltaram a importância } \\
\text { da fisioterapia no PSF, pois por meio da sua } \\
\text { ação preventiva e assistencial os custos e a } \\
\text { demanda no atendimento terciário podem } \\
\text { ser reduzidos. }\end{array}$ & $\begin{array}{l}\text { Recursos e } \\
\text { infraestrutura }\end{array}$ \\
\hline $\begin{array}{l}\text { Rocha, Kretzer, } \\
2009^{(11)}\end{array}$ & $\begin{array}{l}\text { Atendimentos na unidade básica de } \\
\text { saúde, no domicílio ou em espaços } \\
\text { da comunidade. Desenvolvimento de } \\
\text { cuidados terapêuticos, construção de } \\
\text { redes de apoio e ações educativas. }\end{array}$ & $\begin{array}{l}\text { Importância de estratégias que facilitem a } \\
\text { incorporação das ações de reabilitação na } \\
\text { ESF, com responsabilização por parte de } \\
\text { todos os profissionais de saúde por usuários } \\
\text { com deficiências ou incapacidades. }\end{array}$ & $\begin{array}{l}\text { Formação específica } \\
\text { para o trabalho } \\
\text { interdisciplinar }\end{array}$ \\
\hline $\begin{array}{l}\text { Brasil, Brandão, } \\
\text { Silva, Gondim } \\
\text { Filho, } \\
2005^{(12)}\end{array}$ & $\begin{array}{c}\text { Atividades em grupos e } \\
\text { acompanhamento em domicílio de } \\
\text { indivíduos com diversas condições, } \\
\text { desde neurológicas a gineco-obstétricas. }\end{array}$ & $\begin{array}{l}\text { Ênfase a importância do trabalho preventivo } \\
\text { do fisioterapeuta. A atuação do profissional } \\
\text { gera satisfação da população beneficiada } \\
\text { que requer a ampliação do atendimento. }\end{array}$ & $\begin{array}{l}\text { Carência de } \\
\text { profissionais, de } \\
\text { estrutura e de materiais, } \\
\text { desconhecimento } \\
\text { da população e } \\
\text { dos gestores sobre } \\
\text { as funções do } \\
\text { fisioterapeuta }\end{array}$ \\
\hline $\begin{array}{l}\text { Yonamine, } \\
\text { Trelha, } \\
2009^{(13)}\end{array}$ & $\begin{array}{l}\text { Atividades multiprofissionais como a } \\
\text { formação de grupos de saúde e a criação } \\
\text { de espaços de reflexão e promoção da } \\
\text { saúde além de atendimentos individuais } \\
\text { e acompanhamento domiciliar. }\end{array}$ & $\begin{array}{c}\text { Reflexão sobre a reorganização do serviço } \\
\text { de fisioterapia e da demanda reprimida, em } \\
\text { busca de atenção mais humanizada, integral } \\
\text { e de responsabilização dos profissionais } \\
\text { e usuários. A RMSF evidenciou a } \\
\text { importância do trabalho em equipe, devido } \\
\text { à complexidade encontrada na atenção } \\
\text { primária. }\end{array}$ & $\begin{array}{c}\text { Implementação e } \\
\text { fortalecimento de } \\
\text { mudanças na formação } \\
\text { profissional }\end{array}$ \\
\hline $\begin{array}{l}\text { Langoni, } \\
\text { Valmorbida, } \\
\text { Resende, } \\
2012^{(14)}\end{array}$ & $\begin{array}{l}\text { Orientações e atendimentos individuais, } \\
\text { semanais, nas unidades, a usuários } \\
\text { com necessidade de acompanhamento; } \\
\text { dificuldade de locomoção; atraso no } \\
\text { desenvolvimento neuropsicomotor na } \\
\text { infância; alterações osteomusculares; } \\
\text { incontinência urinária ou alterações } \\
\text { respiratórias. }\end{array}$ & $\begin{array}{l}\text { Iniciativa bem-sucedida. Redução de } \\
\text { sintomas, do uso e da quantidade de } \\
\text { medicamentos, o que pode reduzir custos } \\
\text { públicos e individuais. Os atendimentos } \\
\text { foram mais resolutivos para usuários que } \\
\text { seguiram as orientações, reforçando a } \\
\text { importância da educação em saúde através } \\
\text { do estímulo ao autocuidado e da co- } \\
\text { responsabilização pela saúde. }\end{array}$ & $\begin{array}{l}\text { Necessidade de } \\
\text { mudanças na formação } \\
\text { profissional }\end{array}$ \\
\hline $\begin{array}{l}\text { Figueiredo, } \\
\text { Baracho, Vaz, } \\
\text { Sampaio, } \\
2012^{(15)}\end{array}$ & $\begin{array}{l}\text { Educação de funcionárias de unidade } \\
\text { básica de saúde acerca da atenção } \\
\text { fisioterapêutica na incontinência } \\
\text { urinária para que pudessem, com maior } \\
\text { propriedade, encaminhar usuárias ao } \\
\text { serviço. }\end{array}$ & $\begin{array}{l}\text { O programa instrumentalizou as } \\
\text { funcionárias a desenvolver o autocuidado } \\
\text { e a identificar necessidades de assistência } \\
\text { às usuárias. Reflexão sobre a educação } \\
\text { profissional nos serviços de saúde, } \\
\text { particularmente na atenção básica. }\end{array}$ & $\begin{array}{l}\text { Desconhecimento do } \\
\text { trabalho da fisioterapia } \\
\text { na incontinência } \\
\text { urinária }\end{array}$ \\
\hline
\end{tabular}

NASF: Núcleo de Apoio à Saúde da Família. PSF: Programa Saúde da Família. ESF: Estratégia Saúde da Família. RMSFC: Residência Multiprofissional em Saúde da Família e Comunidade. 
Foram incluídos na revisão 7 estudos, caracterizados conforme ano de publicação, cidade onde foi desenvolvido, tipo de equipe da atenção primária em que o fisioterapeuta desenvolvia as atividades, número de fisioterapeutas participantes e público-alvo das ações.

O Quadro I apresenta as características dos estudos incluídos na revisão integrativa.

De acordo com as variáveis selecionadas para análise, apresentadas no Quadro II, as atividades enfatizaram atenção individual e coletiva tanto em nível preventivo quanto de reabilitação junto a diferentes públicos e apresentaram resultados satisfatórios com impacto positivo na saúde e redução de custos individuais e coletivos. No entanto, enfrentam desafios a sua realização como o número insuficiente de profissionais, a falta de recursos e infraestrutura, a dificuldade de trabalho em equipe e a necessidade de mudanças na formação profissional.

\section{DISCUSSÃO}

As atividades desenvolvidas pela fisioterapia no contexto da atenção primária enfatizam atenção individual e coletiva tanto em nível preventivo quanto de reabilitação junto a diferentes públicos. Constatou-se que os resultados das ações foram satisfatórios. No entanto, foram elencadas dificuldades ao trabalho desenvolvido como o número insuficiente de profissionais, a falta de recursos e infraestrutura, a dificuldade de trabalho em equipe e a necessidade de mudanças na formação profissional.

Apesar de não terem sido aplicadas durante a busca restrições quanto a local ou ano de publicação dos artigos, todos eles tratam de experiências desenvolvidas em municípios brasileiros nos últimos dez anos. Destaca-se que neste período ocorreu o desenvolvimento de estratégias que possibilitaram a efetiva implantação da atenção básica no país. Tais estratégias tiveram como referências iniciais o Programa Saúde da Família (PSF) que teve início em 1994 e o Núcleo de Apoio à Saúde da Família (NASF), que atua desde $2008^{(5)}$.

Estas equipes desenvolvem atividades multiprofissionais junto às comunidades. No entanto, nem sempre contemplam a fisioterapia em sua constituição o que pode ser evidenciado pelo pequeno número de profissionais que integram as equipes dos estudos analisados.

A fisioterapia não consta como profissão participante do PSF cuja equipe é formada por médicos, enfermeiros, cirurgiões-dentistas, auxiliares de enfermagem e agentes comunitários de saúde ${ }^{(9)}$. Entretanto, algumas equipes do PSF devido à demanda a que são submetidas de acordo com as necessidades locais do território de atuação incluem fisioterapeutas em suas equipes o que justifica, por exemplo, a inserção de fisioterapeutas no PSF de Londrina ${ }^{(10)}$, São Paulo(11) $^{(1)}$ e Sobral ${ }^{(12)}$.

Apenas os NASF contemplam o profissional fisioterapeuta em sua equipe, porém, não garantem sua plena inserção na atenção primária, pois a inclusão do profissional fica a cargo do gestor municipal que o elenca como membro conforme julgue as necessidades locais ${ }^{(5)}$.

Nesse sentido, por tratar-se de processo ainda em construção a inserção do fisioterapeuta no nível primário de atenção é experimentada em diversas equipes de trabalho. Além de equipes de PSF e NASF os fisioterapeutas compõem também equipes de Residência Multiprofissional em Saúde da Família (RMSF), atuantes nas Unidades Básicas de Saúde.

As RMSF, criadas pelo Ministério da Saúde em 2002, objetivam melhor qualificação de profissionais de saúde para atuação na atenção básica bem como o fortalecimento da equipe multiprofissional ${ }^{(13,14)}$. Dessa forma, agregam aos saberes técnicos adquiridos na graduação a sensibilidade para lidar com a comunidade considerando suas peculiaridades e necessidades.

Um estudo aponta que na residência multiprofissional o fisioterapeuta participa de construções coletivas por meio de reuniões com preceptores, tutores e residentes de outras áreas da saúde, em decisões conjuntas, estabelecem metas a serem atingidas em relação à gestão e ao cuidado das populações sob sua responsabilidade ${ }^{(13)}$.

Além da diversidade de equipes onde o fisioterapeuta desenvolve atividades o público-alvo das intervenções também foi diversificado. Alguns estudos direcionaramse a ações de prevenção e promoção $0^{(5,15)}$, enquanto outros abordaram os dois níveis de atenção à saúde, mesclando atendimentos clínicos individuais com ações educativas direcionadas a grupos específicos ${ }^{(10,14)}$.

Todo esse escopo de atividades é contemplado pelo fisioterapeuta que pode atuar na atenção primária promovendo assistência preventiva e orientações para responsabilização dos usuários bem como na atenção secundária e terciária por meio de tratamento e reabilitação de agravos à saúde ${ }^{(14)}$. Além disso, a diversidade de público e faixa etária demonstra a importância da fisioterapia em todos os ciclos da vida ${ }^{(12,13)}$.

Foram predominantes os atendimentos individuais e as palestras educativas. Este achado confirma a dualidade ainda presente na atividade da fisioterapia junto ao nível primário que embora tente ajustar-se à prevenção e promoção de saúde é redirecionada para a atenção terciária devido à demanda existente.

Observou-se ainda que na atenção primária o fisioterapeuta realiza visita domiciliar e orientação a cuidadores. $\mathrm{O}$ atendimento domiciliar possibilita 
conhecimento da realidade social, econômica, cultural e familiar do paciente o que permite melhor conduta e orientação quanto aos cuidados e a importância da continuidade do tratamento ${ }^{(10)}$.

Destaca-se que as visitas domiciliares devem ter uma abordagem familiar, não se centrando apenas no indivíduo acometido por alguma doença, mas promovendo responsabilização de todos os membros, buscando soluções mais eficientes que não atrelem as condições de saúde à dependência do profissional e empoderem os indivíduos para que sejam responsáveis pela produção de saúde para si e para o contexto coletivo em que vivem ${ }^{(2,14)}$.

Ações direcionadas a grupos foram constantes no trabalho da fisioterapia na atenção primária. Dentre os principais públicos, destacam-se os grupos de gestantes, grupos de postura, grupos de prevenção de inaptidão em hanseníase, grupo de idosos, grupos de trabalhadores, grupos de diabéticos e hipertensos e de usuários de prótese $\mathrm{e}$ órtese $\mathrm{e}^{(16)}$. As atividades também contemplaram grupos específicos como idosos ${ }^{(5)}$, pacientes com incontinência urinária $^{(14,15)}$ e deficientes ${ }^{(11)}$.

Outra atividade destacada foi a educação profissional por meio de ações para promoção de saúde e qualidade de vida de trabalhadoras em saúde ${ }^{(15)}$. Foram fornecidas informações direcionadas a demanda para assistência fisioterapêutica de pacientes com incontinência urinária nos serviços de atenção básica.

Quanto ao local de realização das atividades, os atendimentos de reabilitação ocorreram não só nas UBS e domicílios, mas também em espaços comunitários mais próximos das residências, facilitando o deslocamento do usuário e o acesso ao serviço de saúde ${ }^{(11)}$.

Todos os trabalhos ressaltaram a importância das atividades desenvolvidas pela fisioterapia na atenção primária à saúde, mostrando resultados satisfatórios da atuação deste profissional. A literatura demonstra que além da prevenção, a fisioterapia no nível primário facilita o acesso dos usuários que têm nela alternativa terapêutica de modo a promover qualidade de vida à população já acometida por algum agravo ${ }^{(14)}$.

Na atenção primária, a Fisioterapia constitui também um primeiro contato de modo a realizar o encaminhamento de casos que apresentem necessidade a serviços especializados. Sendo assim, reafirmam a necessidade da atenção à saúde de pessoas com deficiências e incapacidades, que deve ocorrer em diferentes níveis do sistema de saúde conforme prevê o Sistema Único de Saúde (SUS). No entanto, essa assistência encontra-se, predominantemente, vinculada às ações de nível secundário ou terciário, fator que dificulta $\mathrm{o}$ acesso, impondo limites objetivos à integralidade da assistência a esses usuários ${ }^{(11)}$.
Assim, o processo de trabalho encontra dificuldades à sua realização. Dentre as citadas estão o número insuficiente de profissionais, a falta de recursos e infraestrutura, a dificuldade de trabalho em equipe, o desconhecimento da atividade do fisioterapeuta por parte dos demais profissionais de saúde e a necessidade de mudança na formação do profissional ${ }^{(5,10-14)}$.

Apesar da crescente expansão, na atenção primária, da assistência prestada pela fisioterapia ainda persiste na formação a ênfase a práticas curativas e reabilitadoras bem como o estigma reabilitador entendido por outros profissionais da saúde ${ }^{(12,17)}$.

Também por não estar oficialmente incluída na ESF a fisioterapia não dispõe de infraestrutura adequada na unidade básica de saúde ${ }^{(10)}$. Para tanto, é importante a tomada de medidas por parte dos gestores, de forma a garantir recursos para a atuação do fisioterapeuta na atenção primária, melhorando, assim, a assistência prestada à população $0^{(5)}$.

Nesse sentido, reafirma-se a importância do conhecimento das atividades desenvolvidas pelos demais integrantes da equipe multiprofissional de modo a interagir através das peculiaridades relativas a cada área e construir ações interdisciplinares que contemplem os indivíduos e coletividades de maneira integral.

Aponta-se como limitação do estudo a falta de detalhamento de algumas das atividades relatadas nos artigos analisados, o que não permitiu investigação mais aprofundada. No entanto, a análise evidenciou características gerais que possibilitaram conhecer um panorama da realidade da fisioterapia na atenção primária à saúde.

\section{CONCLUSÃO}

As atividades desenvolvidas pela fisioterapia na atenção primária à saúde contemplam diversos públicos por meio de diferentes estratégias. A plena atuação do profissional neste nível de atenção é dificultada devido às condições físicas e econômicas por vezes inadequadas a realização das ações, o desconhecimento por parte da equipe acerca das atividades desenvolvidas fisioterapia no âmbito da atenção básica, o número reduzido de profissionais atuantes no nível primário e a ênfase curativa e reabilitadora ainda predominante na graduação.

Foi possível observar que as ações desenvolvidas vêm apresentando bons resultados e demonstram assim a importância da fisioterapia na atenção primária à saúde o que contribui para a difusão deste campo de atuação.

Nesse sentido, recomenda-se o acompanhamento das atividades desenvolvidas e divulgação delas por meio 
de produções científicas para fomento das experiências e burilamento das ações de modo a proporcionar a efetiva inclusão da fisioterapia nesse nível de cuidado.

\section{REFERÊNCIAS}

1. Neves LMT, Aciole GG. Desafios da integralidade: revisitando as concepções sobre o papel do fisioterapeuta na equipe de Saúde da Família. Interface Comun Saúde Educ. 2011;15(37):551-64.

2. Aveiro MC, Aciole GG, Driusso P, Oishi J. Perspectivas da participação do fisioterapeuta no Programa Saúde da Família na atenção à saúde do idoso. Ciênc Saúde coletiva. 2011;16(Supl 1):1467-78.

3. Ribeiro CD, Soares MCF. Situações com potencialidade para atuação da fisioterapia na atenção básica no Sul do Brasil. Rev Panam Salud Pública. 2014;36(2):117-23.

4. Oliveira G, Andrade ES, Santos ML, Matos GSR. Conhecimento da equipe de saúde da família acerca da atuação do fisioterapeuta na atenção básica. Rev Bras Promoç Saúde. 2011;24(4):332-9.

5 .Dibai AV Filho, Aveiro MC. Atuação dos fisioterapeutas dos núcleos de apoio à saúde da família entre idosos do município de Arapiraca-AL, Brasil. Rev Bras Promoç Saúde. 2012;25(4):397-404.

6. Andrade AMSM, Queiroz PP. Education in health for the physiotherapist: dilemmas in academia education - a study case. Online Braz J Nurs [Internet]. 2013 [acesso em 2016 Mar 12];12:746-8. Disponível em: http://www.objnursing.uff.br/index.php/nursing/ article $/$ view $/ 4506 \% 2 \mathrm{~A} / \mathrm{pdf}$

7. David MLO, Ribeiro MAGO, Zanolli ML, Mendes RT, Assumpção MS, Schivinski CIS. Proposta de atuação da fisioterapia na saúde da criança e do adolescente: uma necessidade na atenção básica. Saúde Debate. 2013;37(96):120-9.

8. Mendes KDS, Silveira RCCP, Galvão CM. Revisão integrativa: método de pesquisa para incorporação de evidências na saúde e na enfermagem. Texto \& Contexto Enferm. 2008;17(4):758-64.

9. Ribeiro MDA, Bezerra EMA, Costa MS, Castelo Branco CE, Araújo JD Neto, Moreira AKF et al. Avaliação da atuação do Núcleo de Apoio à Saúde da Família. Rev Bras Promoç Saúde. 2014;27(2):224-31.
10. Trelha CS, Silva DW, Lida LM, Fortes MH, Mendes TS. O fisioterapeuta no programa de saúde da família em Londrina (PR). Espaço Saúde. 2007;8(2):20-5.

11. Rocha EF, Kretzer MR. Ações de reabilitação de pessoas com deficiência na estratégia da saúde da família da Fundação Zerbini e Secretaria Municipal de Saúde de São Paulo - Região Sudeste - Sapopemba/ Vila Prudente - período 2000/2006. Rev Ter Ocup. 2009;20(1):59-67.

12. Brasil ACO, Brandão JAM, Silva MON, Gondim VC Filho. O papel do fisioterapeuta do programa saúde da família do município de Sobral - Ceará. Rev Bras Promoç Saúde. 2005;18(1):3-6.

13. Yonanime CY, Trelha CS. O modo de fazer saúde: A fisioterapia na residência multiprofissional em saúde da família em uma unidade básica. Espaço Saúde. 2009;11(1):17-27.

14. Langoni CS, Valmorbida LA, Resende TL. A introdução de atendimentos por fisioterapeutas em unidades da atenção primária em saúde. Rev Bras Promoç Saúde. 2012;25(3):261-70.

15. Figueiredo EM, Baracho SM, Vaz CT, Sampaio RF. Educação de funcionárias de unidade básica de saúde acerca da atenção fisioterapêutica na incontinência urinária: relato de experiência. Fisioter pesqui. 2012;19(2):103-8.

16. Naves CR, Brick VS. Análise quantitativa e qualitativa do nível de conhecimento dos alunos do curso de fisioterapia sobre a atuação do fisioterapeuta em saúde pública. Ciênc Saúde Coletiva. 2011;16(Supl 1):152534.

17. Ferretti F, Nierotka RP, Braghini CC, Teo CRPA, Ferraz L, Fanticelli ML. Physical therapist insertion in the Family Health Strategy team: the users' view. Fisioter Mov. 2015;28(3):485-93.

\author{
Endereço para correspondência: \\ Juliany Marques Abreu da Fonseca \\ Universidade Federal do Piauí - UFPI \\ Centro de Ciências da Saúde (CCS) \\ Avenida Frei Serafim, 2280 \\ Bairro: Centro \\ CEP: 64001-020 - Teresina (PI) - Brasil \\ E-mail: juliany.markes@hotmail.com
}

